\title{
Diagnostic accuracy of contrast-enhanced ultrasound for characterization of kidney lesions in patients with and without chronic kidney disease
}

Emily Hueywen Chang ${ }^{1 *}$, Wui Kheong Chong ${ }^{4,5}$, Sandeep Kumar Kasoji ${ }^{7}$, Julia Rose Fielding ${ }^{2,5}$, Ersan Altun ${ }^{5}$, Lee B. Mullin ${ }^{7}$, Jung In Kim ${ }^{6}$, Jason Peter Fine ${ }^{6}$, Paul Alexander Dayton ${ }^{7}$ and Wendy Kimryn Rathmell ${ }^{3,8}$

\begin{abstract}
Background: Patients with chronic kidney disease are at increased risk of cystic kidney disease that requires imaging monitoring in many cases. However, these same patients often have contraindications to contrast-enhanced computed tomography and magnetic resonance imaging. This study evaluates the accuracy of contrast-enhanced ultrasound (CEUS), which is safe for patients with chronic kidney disease, for the characterization of kidney lesions in patients with and without chronic kidney disease.

Methods: We performed CEUS on 44 patients, both with and without chronic kidney disease, with indeterminate or suspicious kidney lesions (both cystic and solid). Two masked radiologists categorized lesions using CEUS images according to contrast-enhanced ultrasound adapted criteria. CEUS designation was compared to histology or follow-up imaging in cases without available tissue in all patients and the subset with chronic kidney disease to determine sensitivity, specificity and overall accuracy.

Results: Across all patients, CEUS had a sensitivity of $96 \%$ (95\% Cl: 84\%, 99\%) and specificity of 50\% (95\% Cl: 32\%, 68\%) for detecting malignancy. Among patients with chronic kidney disease, CEUS sensitivity was $90 \%$ (95\% Cl: 56\%, 98\%), and specificity was 55\% (95\% Cl: 36\%, 73\%).

Conclusions: CEUS has high sensitivity for identifying malignancy of kidney lesions. However, because specificity is low, modifications to the classification scheme for contrast-enhanced ultrasound could be considered as a way to improve contrast-enhanced ultrasound specificity and thus overall performance. Due to its sensitivity, among patients with chronic kidney disease or other contrast contraindications, CEUS has potential as an imaging test to rule out malignancy.
\end{abstract}

Trial registration: This trial was registered in clinicaltrials.gov, NCT01751529.

Keywords: Contrast, Ultrasound, Contrast-enhanced ultrasound, Kidney, Kidney lesion, Chronic kidney disease

\footnotetext{
*Correspondence: emily_chang@med.unc.edu

'University of North Carolina, 7024 Burnett Womack, CB 7155, Chapel Hill, NC

27599, USA

Full list of author information is available at the end of the article
}

(c) The Author(s). 2017 Open Access This article is distributed under the terms of the Creative Commons Attribution 4.0 International License (http://creativecommons.org/licenses/by/4.0/), which permits unrestricted use, distribution, and reproduction in any medium, provided you give appropriate credit to the original author(s) and the source, provide a link to the Creative Commons license, and indicate if changes were made. The Creative Commons Public Domain Dedication waiver (http://creativecommons.org/publicdomain/zero/1.0/) applies to the data made available in this article, unless otherwise stated. 


\section{Background}

The incidence of kidney cancer is increasing with over 60,000 new kidney cancer diagnoses projected for 2017. Kidney cancer is also deadly. Over 14,000 associated deaths are expected in 2017 [1]. If detected early, kidney cancer can be treated effectively with surgery alone. Benign cystic lesions are common, accounting for up to $30 \%$ of all identified lesions [2]. Distinguishing malignant kidney neoplasms from benign lesions is crucial to determining appropriate treatment. Diagnostic options include biopsy $[3,4]$ or interval imaging over months or years [5-7]. Cystic lesions can be characterized with contrast-enhanced computed tomography (CT) or magnetic resonance (MR) imaging [8] using the Bosniak criteria $[9,10]$.

However, Bosniak classification requires contrast enhancement which may pose risk in some patients with compromised kidney function. Risks associated with iodinated contrast agents used with $\mathrm{CT}$ imaging include further impairment in kidney function for those with advanced stages of chronic kidney disease (CKD). Use of gadolinium-based contrast agents in this population also poses a rare but serious risk of nephrogenic sclerosing fibrosis. Incidence of this disease has virtually disappeared with more stringent screening prior to gadolinium exposure and use of agents with better risk profiles. However, gadolinium deposition in various organs is now being postulated even in patients with normal kidney function, although the clinical significance is not yet known $[11,12]$. Patients with impaired kidney function experience prolonged exposure to gadolinium since it is cleared primarily by the kidneys and thus have an increased risk of gadolinium deposition [13]. Over 40 million people in the United States have chronic kidney disease (CKD), and this population is rapidly growing (USRDS 2016). These patients have substantially increased risk for the development of kidney cancer [14-16]. However, the typical screening tools of contrast-enhanced CT and MR are often contraindicated in patients with advanced stages of CKD. In patients with moderate stages of CKD, kidney-protective measures can be taken but add additional steps, can be cumbersome and do not entirely eliminate risk. Even in these populations, if a patient has a high-risk lesion, the benefits of a contrast CT or MR study may outweigh the risks from contrast exposure. For patients with lower or moderate risk lesions, the risk of contrast-enhanced CT or MR may not be worth the benefit. Radiologic options for this population are limited [17]. Non-contrasted surveillance imaging is often performed, but this is inferior to contrast enhanced studies for lesion characterization.

A potential alternative imaging modality for evaluating kidney lesions is contrast-enhanced ultrasound (CEUS). CEUS has been used to characterize indeterminate lesions in multiple organs $[18,19]$, including the kidneys [20-27].
CEUS has the ability to detect vascularization as the microbubble contrast agent remains purely intravascular $[28,29]$. A unique aspect of CEUS is its ability to image contrast dynamics in real time [30,31]. US contrast agents have a low serious adverse event rate of $0.006-0.009 \%$, consisting primarily of anaphylactoid reactions that resolve when the contrast is cleared and typically do not necessitate hospitalization [32, 33]. Less serious side effects that are also rare and typically transient, resolving when the contrast agent is cleared, include headache, dizziness, flushing, nausea, flank pain and chest pain. An additional benefit setting them apart from other contrast agents is that they are excreted by exhalation through the lungs and are therefore not nephrotoxic and safe for patients with kidney disease. Despite the potential for CEUS as a diagnostic option among individuals with CKD, few studies have evaluated CEUS in this population, although interest is increasing $[34,35]$. In this exploratory pilot study, we sought to investigate the diagnostic accuracy of CEUS for detecting enhancement of kidney lesions, and thus risk of malignancy, in populations with and without CKD and subsequently determine if this imaging modality has potential to be an alternative screening tool in patients with contraindications to contrast CT or MR. We hypothesized that CEUS has sensitivity comparable to contrast-enhanced CT or MR among individuals with and without CKD.

\section{Methods}

\section{Study design and participants}

We performed a prospective imaging study of CEUS in patients with kidney lesions identified by prior imaging (ultrasound, CT or MR) obtained as part of routine clinical care. This study was performed in compliance with the policies related to the use of human subjects of the Biomedical Institutional Review Board. All procedures performed were in accordance with the ethical standards of the institutional research committee and with the 1964 Helsinki declaration and its later amendments or comparable ethical standards. Informed consent was obtained from all individual participants included in the study. This pilot study was designed to generate needed data to allow for accurate power calculations in future studies. The numbers of true positives and true negatives were random and not fixed by the study design. The total sample size of 48 was chosen based on based on estimated ability to recruit 2-3 patients a month over a 2 -year time period. Any patient meeting inclusion/exclusion criteria was offered participation in the study. Inclusion criteria were: 1) eligibility for nephrectomy or ablative therapy based on identification of a kidney lesion on prior imaging, or having CKD and an incompletely characterized kidney lesion on prior imaging; and 2) the 
ability to provide informed consent and comply with protocol requirements.

Exclusion criteria included: 1) active cardiac disease, including class IV congestive heart failure, unstable angina, severe arrhythmia, myocardial infarction within 14 days prior to the study and uncontrolled blood pressure $(>150$ / $90 \mathrm{mmHg}$ ); 2) severe pulmonary hypertension or adult respiratory distress syndrome; 3) hypersensitivity to the Definity (Perflutren lipid) US contrast agent; 4) critical illness or intensive care unit status; 5) right-to-left cardiac shunt; 6) unstable neurologic disease within 3 months; 7) invasive kidney procedure between time of lesion identification and CEUS; 8) mental illness or drug abuse; and 9) pregnancy or lactation. Patients were recruited from Urology and Nephrology clinics from July 2013 to November 2014. After obtaining informed written consent, patients underwent CEUS per a standard study protocol with low mechanical index (MI) (0.19) imaging.

Because the Bosniak criteria $[9,10]$ were not designed for ultrasound, for cystic lesions, we applied an adapted Bosniak criteria to CEUS, including both B-mode and CEUS images, (Additional file 1: Table S1) by substituting "internal echogenicity" within a cyst for "high-attenuation". High attenuation within a cyst is an indicator of proteinaceous or hemorrhagic content, which appears on US as internal echogenicity. Lesions were categorized as solid or Bosniak I-IV by CEUS. In primary analyses, the CEUS diagnosis was compared to the reference standard of tissue diagnosis (malignant/benign). CEUS Bosniak I, II and IIF lesions were considered negative since these are generally managed non-surgically; CEUS Bosniak III, IV and solid lesions were considered positive since these are generally managed surgically. In secondary analyses, we used a reference standard of tissue diagnosis or follow-up imaging (obtained 12-26 months after CEUS). The follow-up interval and imaging modality was determined by the individual's doctor. Follow-up imaging modalities included standard B-mode US, contrast $\mathrm{CT}$ and contrast MR. For patients with more than one follow-up imaging examination, the last available examination was used for analysis. Stable lesions were considered negative. A worsening of concerning lesion characteristics (septations, calcifications, mural thickness, irregularity or nodules) was considered positive. The reference standard of follow-up imaging was used because tissue diagnosis was not feasible in many patients given the risks associated with surgery and biopsy.

In separate analyses among patients who had a clinical contrast-enhanced CT or MR $(n=25)$, CEUS Bosniak classification was compared to CT/MR Bosniak classification, by the same blinded readers, to determine inter-modality agreement. To determine inter-reader agreement, answers to lesion characteristic questions
(Additional file 1: Table S1) were compared across readers for CEUS and CT/MR studies. The median time interval between CEUS and CT/MR was 29 days (interquartile range: $20-44)$.

\section{Imaging procedure and analysis}

CEUS was performed with the Siemens Acuson Sequoia 512 (Siemens, Mountain View, CA, USA) with contrast specific software using a $4 \mathrm{C} 1$ abdominal transducer. A standard MI of 1.9 was used for B-mode imaging and a low MI of 0.19 was used for all CEUS clips. The MI is a metric used to describe potential bioeffects caused by ultrasound, specifically cavitation bioeffects. A higher MI indicates increased likelihood of bioeffects. Typical B-mode ultrasound imaging uses an MI of 1.9. Low MI (0.19) is used in CEUS to avoid disruption of microbubbles. Scanning was performed by registered sonographers trained in contrast imaging. Lesions were located with B-mode ultrasound. For patients with multiple lesions, the most complex lesion as designated on prior imaging was chosen for CEUS imaging. The transducer was then positioned over the lesion so that the imaging plane included part of the normal kidney parenchyma. The contrast agent, Perflutren Lipid microspheres (Definity $^{\mathrm{R}}$, Lantheus, North Billerica, MA), was prepared as a bolus injection, as described in the package insert instructions. The total contrast volume administered was based on weight, $0.50 \mathrm{~mL}$ for $<125 \mathrm{lb}$., $0.65 \mathrm{~mL}$ for 125-185 lb., and $1.0 \mathrm{~mL}$ for $>185 \mathrm{lb}$. This contrast was diluted in saline to a final volume of $5 \mathrm{~mL}$ and injected over $15 \mathrm{~s}$, followed by a $5 \mathrm{~mL}$ saline flush. Lesions were imaged for $3 \mathrm{~min}$ after contrast injection.

Images were de-identified and interpreted by two radiologists blinded to lesion diagnosis and to each other's reads. A custom graphical user interface (GUI) developed in MATLAB (MathWorks, Natick, MA) displayed deidentified CEUS and B-mode clips accompanied by questions related to lesion characteristics (Additional file 2: Figure S1). Two abdominal radiologists (identified as Reader 1 and Reader 2 with 20 and 15 years' experience in ultrasound imaging, respectively) not involved in image acquisition independently performed the blinded study reads. Both readers participated in a preliminary training session and performed CEUS radiology literature review, particularly from experienced centers such as the University of Calgary, and international ultrasound societies [36]. Readers used both B-mode and CEUS images to answer lesion characteristic questions, classify the lesions as solid or cystic, and if cystic, apply the adapted Bosniak criteria (Additional file 1: Table S1). CEUS enhancement was determined by the subjective determination of the appearance of contrast on the image. The diagnosis based on the B-mode and CEUS images is designated "CEUS diagnosis". The same radiologists reviewed the 
initial contrast-enhanced CT and MR images when available and answered the same questions. A 3-month delay between CEUS and CT/ MR readings was instituted to prevent recall.

Contrast-enhanced CTs were acquired with a 16- to 64-slice MDCT scanner. A non-contrasted scan was first obtained. $100 \mathrm{~mL}$ of Iohexol $755 \mathrm{mg} / \mathrm{ml}$ (Omnipaque 350 - GE Healthcare, Milwaukee, WI) was administered intravenously at 3-4 mL/s. MRs were performed on 1.5 Avanto and $3 \mathrm{~T}$ Trio (Siemens, Iselin, NJ, USA). $10 \mathrm{ml}$ of Gadobenate Diglumine (Multihance, Bracco Diagnostics, Monroe Township, NJ) was administered intravenously. For details about the CEUS, CT and MR imaging techniques, see Additional file 3.

\section{Statistical analysis}

For the primary analysis, CEUS diagnosis was compared to the reference standard of tissue diagnosis. For secondary analyses, CEUS diagnosis was compared to tissue diagnosis or follow-up imaging diagnosis. Sensitivity, specificity and overall accuracy (number of CEUS correct diagnoses/total number of lesions) were calculated for each reader separately, with $95 \%$ confidence intervals using the exact Pearson-Clopper method. Reader data were considered separately and combined, with measures calculated using generalized estimating equations [37], along with asymptotic $95 \%$ confidence intervals under a working independence assumption between readers.

Performance measures (sensitivity, specificity and overall accuracy) were also determined for the subset of patients with CKD, with analyses analogous to those used in the full cohort. These exploratory secondary analyses were stratified according to CKD severity: early CKD (GFR $\geq 30 \mathrm{~mL} / \mathrm{min}$ ) and advanced CKD (GFR $<30 \mathrm{~mL} / \mathrm{min}$, including patients on dialysis or the native kidney of a patient with a kidney transplant). Because of small sample sizes, the current study was underpowered to demonstrate statistically significant differences in diagnostic accuracy between late and early CKD groups. To detect the observed differences in total accuracy of 0.8 versus 0.5 at significance level 0.05 using a 2 -sided test of two proportions, 40 subjects in each CKD group would be needed for $80 \%$ power and 50 subjects per group for $90 \%$ power.

Additional exploratory subgroup secondary analyses considered accuracy across patients with a priori-designated co-morbid conditions (hypertension, diabetes mellitus, cardiovascular disease and obesity) and across lesion sizes $(<3$ vs. $\geq 3 \mathrm{~cm})$. Fisher's exact test was used to calculate statistical significance. Agreement between readers was calculated using sample proportions. All analyses were conducted in SAS version 9.4 (Cary, NC). Two-tailed $p$-values of $<0.05$ were used to indicate statistical significance.

\section{Results}

\section{Patient and lesion characteristics}

A flowchart of patient recruitment is shown in Fig. 1. Of the 48 patients who underwent CEUS examination, 2 were excluded due to poor technical quality. Of the 46 patients that received CEUS classification, 2 were excluded due to lack of follow-up. One died of unrelated cause (meningitis), and another patient with a suspicious lesion recommended for nephrectomy opted for surveillance, but was lost to follow-up. A total of 44 patients had interpretable CEUS imaging with one lesion examined per patient and either tissue diagnosis or follow-up imaging results available. A summary of patient and lesion characteristics is displayed in Table 1 . Mean patient age was 56 , with $70 \%(31 / 44)$ being male. The most common co-morbidities were hypertension $(68 \%, 30 / 44)$, hyperlipidemia $(50 \%, 22 / 44)$ and obesity $(50 \%, 22 / 44)$. Of the 44 patients, 25 had CKD, including 7 patients receiving dialysis and 3 who had received a kidney transplant. All 3 had functioning transplants. Patients with CKD were more likely to be older and have hypertension and cardiovascular disease.

The most common initial study for patients without CKD was contrast CT $(n=15)$ followed by contrast MR $(n=5)$. The most common initial study for patients with CKD was a conventional, non-contrasted ultrasound $(n=17)$, followed by non-contrasted CT $(n=8)$. Patients with CKD were more likely to have bilateral lesions with smaller diameters of individual lesions. The average diameter based on initial imaging study was $3.51 \mathrm{~cm}$ in nonCKD patients and $3.09 \mathrm{~cm}$ in CKD patients.

Of the 44 patients in the study cohort (Fig. 1), 23 underwent surgery/biopsy and 21 were followed with serial imaging. Of the 23 surgical patients, 22 had nephrectomy and 1 had fine needle aspiration showing angiomyolipoma. The primary reference standard of histologic diagnosis categorized 2 lesions as benign and 21 as malignant. The observed malignancy subtypes (clear cell, papillary, and chromophobe) followed patterns observed in prior studies [38]. More papillary renal cell carcinomas were observed in patients with CKD compared to patients without CKD, as has been previously observed [39]. The secondary reference standard of tissue diagnosis or diagnosis on follow-up imaging categorized 21 lesions as benign ( 2 by histology and 19 by imaging) and 23 as malignant ( 21 by histology and 2 by imaging). The mean follow-up period was 20 months and ranged from 12 to 26 months.

\section{CEUS accuracy}

Accuracy was defined as the number of CEUS correct diagnoses/total number of lesions. Table 2 displays combined and individual reader accuracy results, including sensitivity, specificity and overall accuracy for the 


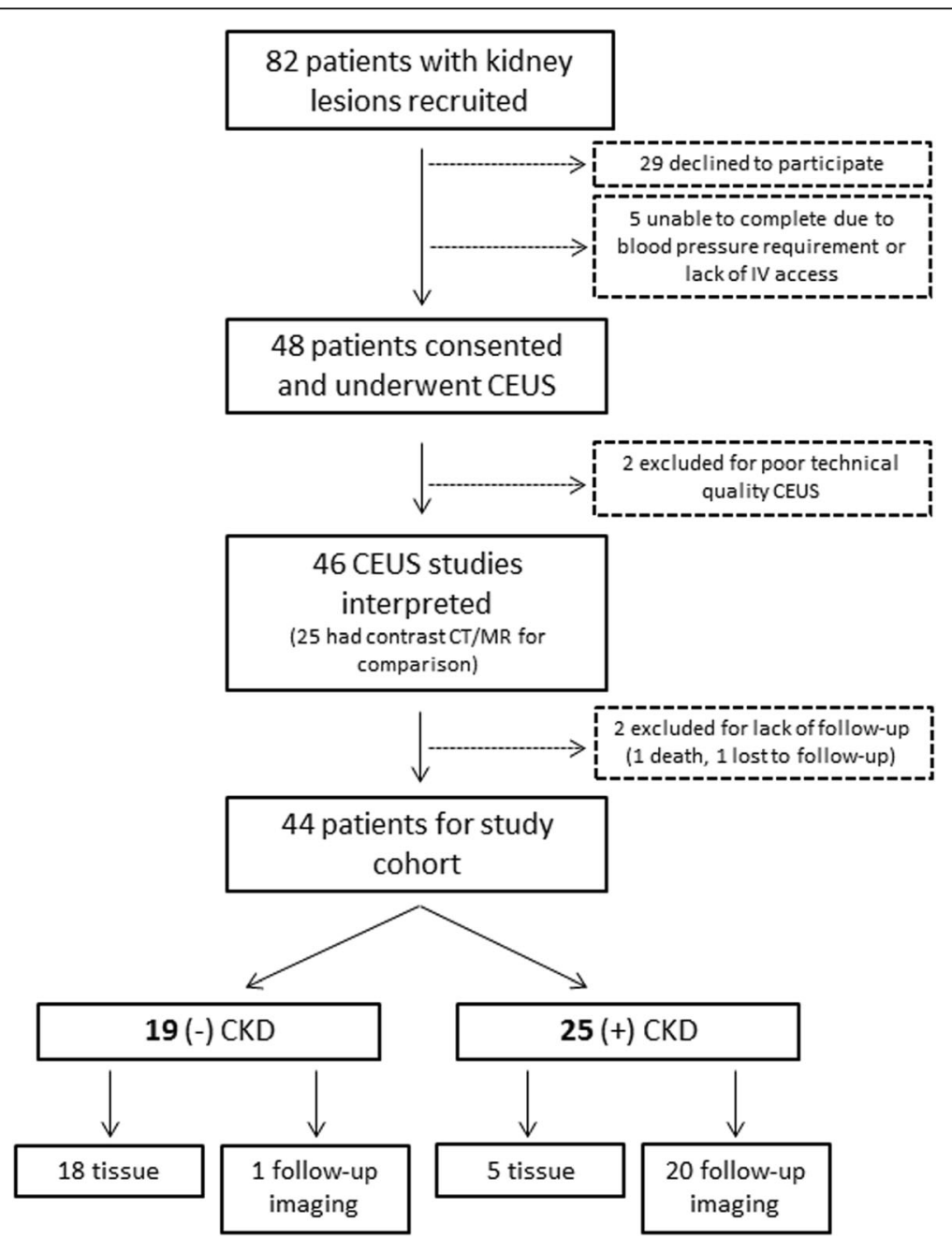

Fig. 1 Patient flowchart. Flowchart of patient recruitment, exclusions and numbers for final analysis

diagnosis of kidney cancer. In the primary analysis using tissue diagnosis as the reference standard, combined overall accuracy was $87 \%$ (95\% CI: 69\%, 95\%). In secondary analyses using tissue or follow-up imaging as the reference standard, combined overall accuracy was $73 \%$ (95\% CI: 59\%, 83\%). The cohort size was inadequate for analyzing CEUS characterization based on histological subtype (clear cell, papillary, or chromophobe).

\section{Inter-reader agreement}

CEUS inter-reader agreement results are shown in Fig. 2. The highest rate of agreement was seen with calcifications (89\%), overall malignant/benign designation (87\%), mural nodules (83\%) and lesion enhancement (80\%). The lowest rate of agreement was with Bosniak class (57\%), septa (63\%) and rim enhancement (65\%). Low rate of Bosniak class agreement was not surprising as there are 6 possible categories (solid, I, II, IIF, III and IV).
CEUS diagnoses compared to contrast $\mathrm{CT} / \mathrm{MR}$ diagnoses CEUS diagnosis was compared to CT/MR diagnosis for 25 patients who had both CEUS and a contrasted CT/ MR. Of the studies that were positive (Bosniak III, IV or solid) by CT/MR, $89 \%$ were also positive by CEUS for reader 1 and $96 \%$ for reader 2 (Table 3 ). Of the studies that were negative (Bosniak I, II and IIF) by CT/MR, $83 \%$ were read as positive by CEUS for reader 1 and $100 \%$ for reader 2 . There was no overlap in confidence intervals for these two groups for either reader. Overall agreement between CT/MR and CEUS diagnoses was $72 \%$ for reader 1 and $92 \%$ for reader 2 . Inter-reader agreement with CT/MR was found to be $81 \%$ for malignant/benign designation.

\section{Accuracy in patients with chronic kidney disease}

Of the 25 patients with CKD, 5 (20\%) had tissue diagnoses. All 5 of these patients had malignant lesions, and 
Table 1 Patient and lesion characteristics

\begin{tabular}{|c|c|c|c|}
\hline & Total $(N=44)$ & $(+) \operatorname{CKD}(n=25)$ & $(-) \operatorname{CKD}(n=19)$ \\
\hline \multicolumn{4}{|l|}{ Age (years) } \\
\hline Mean \pm S.D. & $56 \pm 14$ & $59 \pm 13$ & $51 \pm 14$ \\
\hline Male & $31(70 \%)$ & $18(72 \%)$ & $13(68 \%)$ \\
\hline \multicolumn{4}{|l|}{ Co-morbid conditions } \\
\hline Hypertension & $30(68 \%)$ & $20(80 \%)$ & $10(53 \%)$ \\
\hline Diabetes & $10(23 \%)$ & $5(20 \%)$ & $5(26 \%)$ \\
\hline Hyperlipidemia & $22(50 \%)$ & $13(52 \%)$ & $9(47 \%)$ \\
\hline Cardiovascular disease & $9(20 \%)$ & $8(32 \%)$ & $1(5 \%)$ \\
\hline History of renal cell carcinoma & $3(7 \%)$ & $2(8 \%)$ & $1(5 \%)$ \\
\hline Obesity $^{a}$ & $22(50 \%)$ & $11(44 \%)$ & $11(58 \%)$ \\
\hline \multicolumn{4}{|l|}{ Initial Study ${ }^{\mathrm{b}}$} \\
\hline Non-contrast CT & $11(25 \%)$ & $8(32 \%)$ & $3(16 \%)$ \\
\hline Contrast CT & $20(45 \%)$ & $5(20 \%)$ & $15(79 \%)$ \\
\hline Non-contrast MR & $1(2 \%)$ & 0 & $1(5 \%)$ \\
\hline Contrast MR & $6(14 \%)$ & $1(4 \%)$ & $5(26 \%)$ \\
\hline Conventional US & $17(39 \%)$ & $17(68 \%)$ & 0 \\
\hline \multicolumn{4}{|l|}{ Laterality of lesion } \\
\hline Right & 19 (43\%) & $12(48 \%)$ & $7(37 \%)$ \\
\hline Left & $20(45 \%)$ & $9(36 \%)$ & $11(58 \%)$ \\
\hline Bilateral & $5(11 \%)$ & $4(16 \%)$ & $1(5 \%)$ \\
\hline \multicolumn{4}{|l|}{ Laterality of imaging } \\
\hline Right & $21(48 \%)$ & $14(56 \%)$ & $7(37 \%)$ \\
\hline Left & $23(52 \%)$ & $11(44 \%)$ & $12(63 \%)$ \\
\hline \multicolumn{4}{|l|}{ Diameter by imaging $(\mathrm{cm})^{c}$} \\
\hline Mean (range) & $3.27(1.4-7.9)$ & $3.09(1.4-7.9)$ & $3.51(1.4-6.6)$ \\
\hline \multicolumn{4}{|l|}{ Diameter by histology $(\mathrm{cm})^{\mathrm{d}}$} \\
\hline Mean (range) & $\begin{array}{l}3.39(0.4-7) \\
(n=20)\end{array}$ & $\begin{array}{l}2.88(0.4-5.6) \\
(n=4)\end{array}$ & $\begin{array}{l}3.51(1.2-7) \\
(n=16)\end{array}$ \\
\hline \multicolumn{4}{|l|}{ Diagnosis } \\
\hline Clear cell RCC & $14(32 \%)$ & $1(4 \%)$ & $13(68 \%)$ \\
\hline Papillary RCC & $5(11 \%)$ & $3(12 \%)$ & $2(11 \%)$ \\
\hline Chromophobe RCC & $2(4 \%)$ & $1(4 \%)$ & $1(5 \%)$ \\
\hline Angiomyolipoma & $1(2 \%)$ & 0 & $1(5 \%)$ \\
\hline Oncocytoma & $1(2 \%)$ & 0 & $1(5 \%)$ \\
\hline Surveillance & $21(48 \%)$ & $20(80 \%)$ & $1(5 \%)$ \\
\hline Stable/benign & 19 & 19 & 0 \\
\hline Progressed/malignant & 2 & 1 & 1 \\
\hline \multicolumn{4}{|l|}{ CKD stage } \\
\hline Non-CKD & 19 (43\%) & 0 & 19 (100\%) \\
\hline CKD ॥ & $2(4 \%)$ & $2(8 \%)$ & 0 \\
\hline CKD III & $9(20 \%)$ & $9(36 \%)$ & 0 \\
\hline
\end{tabular}


Table 1 Patient and lesion characteristics (Continued)

\begin{tabular}{|c|c|c|c|}
\hline CKD IV & $4(9 \%)$ & $4(16 \%)$ & 0 \\
\hline CKD V on dialysis & $7(16 \%)$ & $7(28 \%)$ & 0 \\
\hline Transplant & $3(7 \%)$ & $3(12 \%)$ & 0 \\
\hline \multicolumn{4}{|l|}{ Probable cause of CKD } \\
\hline Hypertensive Nephrosclerosis & & $11(44 \%)$ & \\
\hline Diabetic Kidney Disease & & $4(16 \%)$ & \\
\hline Hypertension/Diabetic Kidney Disease & & $1(4 \%)$ & \\
\hline Polycystic Kidney Disease & & $2(8 \%)$ & \\
\hline Chronic Interstitial Nephritis & & $2(8 \%)$ & \\
\hline Membranous Nephropathy & & $1(4 \%)$ & \\
\hline Focal Segmental Glomerulosclerosis & & $1(4 \%)$ & \\
\hline Medullary Sponge Kidney & & $1(4 \%)$ & \\
\hline NSAID-induced Nephropathy & & $1(4 \%)$ & \\
\hline Lithium Nephrotoxicity & & $1(4 \%)$ & \\
\hline
\end{tabular}

CKD chronic kidney disease, $C T$ computed tomography, MR magnetic resonance imaging, US ultrasound, $R C C$ renal cell carcinoma

${ }^{\mathrm{a}}$ Defined as $\mathrm{BMI} \geq 30$

bercentages add to greater than $100 \%$ as 11 patients had multiple studies prior to CEUS

'By largest dimension

${ }^{\mathrm{d}}$ One sample was resected in multiple pieces with no histologic diameter available

sensitivity of CEUS in these patients was 90\% (95\% CI: $56 \%, 98 \%)$. Compared to tissue or follow-up diagnosis, sensitivity was $90 \%$ (95\% CI: $56 \%, 98 \%)$ and specificity 55\% (95\% CI: 36\%, 73\%). Overall accuracy was 62\% (95\% CI: $44 \%, 77 \%)$. Further analysis by CKD stage showed CEUS sensitivity, specificity and overall accuracy was lower in patients with advanced (stage IV, $\mathrm{V}$, dialysis and transplant) versus early (stage II and III) CKD. The combined overall accuracy for early CKD was 77\% (95\% CI: $48 \%, 93 \%$ ), and $50 \%$ (95\% CI: $29 \%, 71 \%$ ) for advanced CKD. Sensitivity and specificity and individual accuracies for each reader are presented in Table 4.

Numbers were not sufficient to detect significant differences in accuracy between patients with and without hypertension, diabetes, cardiovascular disease and obesity (Additional file 1: Table S2) or to perform analyses on subgroups of solid and cystic lesions (Additional file 1: Table S3). Such analyses were considered exploratory and are presented in Additional file 1: Tables S2 and S3.

Accuracy in patients with chronic kidney disease for small $(<3 \mathrm{~cm})$ vs. large $(\geq 3 \mathrm{~cm})$ lesions

Accuracy was examined for lesions $<3 \mathrm{~cm}(n=23)$ and $\geq 3 \mathrm{~cm}(n=21)$ in diameter in patients with and without CKD. More patients with CKD had smaller lesions (16/23) than larger lesions $(9 / 21)$. For smaller lesions, when combining results from both readers, overall accuracy decreased from $71 \%$ (95\% CI: 33\%, 93\%) for patients with no CKD to 56\% (95\% CI: 34\%, 76\%) for patients with CKD.

Table 2 Accuracy of CEUS compared to tissue diagnosis (primary analysis) and tissue diagnosis or follow-up imaging diagnosis (secondary analysis) $^{a}$

\begin{tabular}{|c|c|c|c|c|c|c|}
\hline & \multicolumn{2}{|c|}{ Reader 1} & \multicolumn{2}{|c|}{ Reader 2} & \multicolumn{2}{|c|}{ Combined readers } \\
\hline & $\begin{array}{l}\text { Tissue diagnosis } \\
(N=23)\end{array}$ & $\begin{array}{l}\text { Tissue or follow-up } \\
\text { imaging } \\
(N=44)\end{array}$ & $\begin{array}{l}\text { Tissue diagnosis } \\
(N=23)\end{array}$ & $\begin{array}{l}\text { Tissue or follow-up } \\
\text { imaging } \\
(N=44)\end{array}$ & $\begin{array}{l}\text { Tissue } \\
\text { diagnosis } \\
(N=23)\end{array}$ & $\begin{array}{l}\text { Tissue or follow-up } \\
\text { imaging } \\
(N=44)\end{array}$ \\
\hline Sensitivity & $\begin{array}{c}90 \% \\
(78 \%, 100 \%) \\
{[19 / 21]}\end{array}$ & $\begin{array}{c}91 \% \\
(80 \%, 100 \%) \\
{[20 / 22]}\end{array}$ & $\begin{array}{c}100 \% \\
(100 \%, 100 \%) \\
{[21 / 21]}\end{array}$ & $\begin{array}{c}100 \% \\
(100 \%, 100 \%) \\
{[22 / 22]}\end{array}$ & $\begin{array}{c}95 \% \\
(83 \%, 99 \%)\end{array}$ & $\begin{array}{c}96 \% \\
(84 \%, 99 \%)\end{array}$ \\
\hline Specificity & $\begin{array}{c}0 \% \\
(0 \%, 0 \%) \\
{[0 / 2]}\end{array}$ & $\begin{array}{c}59 \% \\
(39 \%, 80 \%) \\
{[13 / 22]}\end{array}$ & $\begin{array}{c}0 \% \\
(0 \%, 0 \%) \\
{[0 / 2]}\end{array}$ & $\begin{array}{c}41 \% \\
(20 \%, 61 \%) \\
{[9 / 22]}\end{array}$ & $\begin{array}{c}0 \% \\
(0 \%, 0 \%)\end{array}$ & $\begin{array}{c}50 \% \\
(32 \%, 68 \%)\end{array}$ \\
\hline Overall Accuracy & $\begin{array}{c}83 \% \\
(67 \%, 98 \%) \\
{[19 / 23]}\end{array}$ & $\begin{array}{c}75 \% \\
(62 \%, 88 \%) \\
{[33 / 44]}\end{array}$ & $\begin{array}{c}91 \% \\
(80 \%, 100 \%) \\
{[21 / 23]}\end{array}$ & $\begin{array}{c}70 \% \\
(57 \%, 84 \%) \\
{[31 / 44]}\end{array}$ & $\begin{array}{c}87 \% \\
(69 \%, 95 \%)\end{array}$ & $\begin{array}{c}73 \% \\
(59 \%, 83 \%)\end{array}$ \\
\hline
\end{tabular}

${ }^{a}$ Results presented as accuracy metrics $(95 \% \mathrm{Cl}$ ) and [positive or negative reads/total reads]. CEUS images were read by 2 independent readers. Results were considered per reader and as a combination of the 2 readers. For combined reader result, generalized estimating equations were used considering two readers' values as repeated measurements (or responses) 


\section{Inter-Reader Agreement}

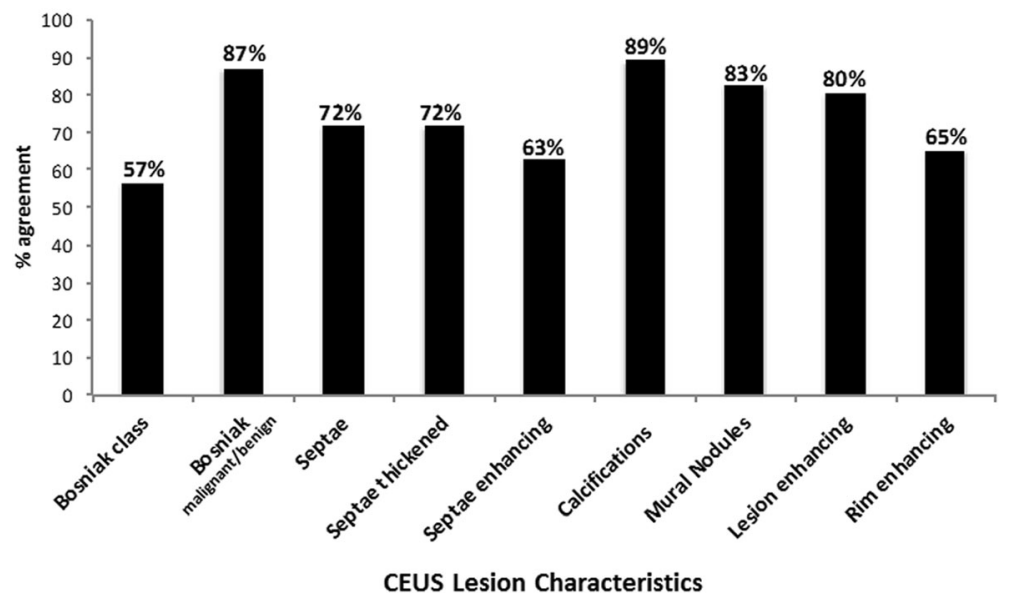

Fig. 2 Inter-reader agreement. The rate of agreement between the two readers was calculated for each individual lesion characteristic, the Bosniak class designation (or solid designation) and the overall designation of malignant or benign based on Bosniak class (Bosniak I, II and IIF considered benign and Bosniak III, IV and solid considered malignant)

For larger lesions, when combining results from both readers, overall accuracy decreased from $96 \%$ (95\% CI: $76 \%, 99 \%)$ for patients with no CKD to $72 \%$ (95\% CI: $46 \%$, 89\%) for patients with CKD. There was no difference in sensitivity for larger lesions but a decrease in sensitivity from $100 \%$ (95\% CI: $100 \%, 100 \%$ ) to $75 \%$ (95\% CI: $32 \%$, 95\%) was obersved in smaller lesions. Results are presented in Additional file 1: Table S4.

\section{Discussion}

Evaluation of kidney lesions by ultrasound has historically been limited by lack of enhancement. Contrastenhanced CT/MR has been the imaging standard. However, iodinated contrast agents may worsen kidney function, and gadolinium agents may cause nephrogenic

Table 3 Agreement between contrast CT/MR and CEUS diagnosis ${ }^{a}$

\begin{tabular}{cccc}
\hline & & Reader 1 & Reader 2 \\
\hline CT/MR+ & CEUS+ & $89 \%$ & $96 \%$ \\
& & $(76 \%, 100 \%)$ & $(87 \%, 100 \%)$ \\
& CEUS- & {$[17 / 19]$} & {$[23 / 24]$} \\
& & $11 \%$ & $4 \%$ \\
CT/MR- & CEUS+ & {$[0 \%, 24 \%)$} & $(0 \%, 13 \%)$ \\
& & $83 \%$ & {$[1 / 24]$} \\
& & $(54 \%, 100 \%)$ & $(100 \%, 100 \%)$ \\
& CEUS- & {$[5 / 6]$} & {$[1 / 1]$} \\
& & $17 \%$ & $0 \%$ \\
& & $(0 \%, 46 \%)$ & $(0 \%, 0 \%)$ \\
& Overall Agreement & {$[1 / 6]$} & {$[0 / 1]$} \\
& & $72 \%$ & $92 \%$ \\
& & {$[18 \%, 90 \%)$} & $(81 \%, 100 \%)$ \\
& & {$[23 / 25]$} \\
\hline
\end{tabular}

${ }^{a}$ Results presented as rate of agreement with $(95 \% \mathrm{Cl})$ and [CEUS interpretation/ CT or MR interpretation] systemic fibrosis in patients with advanced CKD. The primary goal of this pilot study was to explore the detection of microbubble contrast in kidney lesions among patients with and without CKD and to test the accuracy of CEUS for characterizing these lesions as malignant or benign (Fig. 3). We found that the sensitivity of CEUS, whether compared to tissue diagnosis or follow-up imaging, is high and comparable to the reported sensitivities of CT (83-100\%) and MR (81-100\%) [21-24, 40] among patients with and without CKD. High sensitivity provides high certainty that a negative test in an individual means the individual does not have the disease, essentially ruling out malignancy.

Although we found high sensitivity with CEUS, specificity was low. Whether compared to tissue diagnosis or tissue diagnosis/follow-up imaging, specificity of CEUS is lower than reported CT (51-96\%) and MR (71-100\%) specificities [21-24, 40]. Low specificity means a large number of false positives, thus a positive test does not mean the individual has the disease and cannot rule in malignancy very well.

A test with high sensitivity and lower specificity (for example, mammography or CT for lung cancer) is suitable for screening purposes. Benign cystic masses are common in renal insufficiency and distinguishing malignant from benign cysts is a diagnostic challenge. $\mathrm{CT}$ or MR is the current standard, but performing CT/MR on all patients with cystic masses would be prohibitively costly and expose a large number of patients to radiation or potentially nephrotoxic contrast agents. CEUS's ability to exclude malignancy with minimal risk of adverse effects means that the use of CT/MR could be narrowed down to only those patients with a positive CEUS. 
Table 4 Accuracy of CEUS based on severity of CKD compared to the secondary reference standard ${ }^{a}$

\begin{tabular}{|c|c|c|c|c|c|c|}
\hline & \multicolumn{2}{|c|}{ Reader 1} & \multicolumn{2}{|c|}{ Reader 2} & \multicolumn{2}{|c|}{ Combined readers } \\
\hline & $\begin{array}{l}\text { Early CKD } \\
(\mathrm{eGFR} \geq 30 \mathrm{~mL} / \mathrm{min}) \\
(N=11)\end{array}$ & $\begin{array}{l}\text { Advanced CKD } \\
(\mathrm{e} G F R<30 \mathrm{~mL} / \mathrm{min}) \\
(\mathrm{N}=14)\end{array}$ & $\begin{array}{l}\text { Early CKD } \\
(\mathrm{eGFR} \geq 30 \mathrm{~mL} / \mathrm{min}) \\
(\mathrm{N}=11)\end{array}$ & $\begin{array}{l}\text { Advanced CKD } \\
(\mathrm{e} G F R<30 \mathrm{~mL} / \mathrm{min}) \\
(N=14)\end{array}$ & $\begin{array}{l}\text { Early CKD } \\
(\mathrm{eGFR} \geq 30 \mathrm{~mL} / \mathrm{min}) \\
(\mathrm{N}=11)\end{array}$ & $\begin{array}{l}\text { Advanced CKD } \\
(\mathrm{e} G F R<30 \mathrm{~mL} / \mathrm{min}) \\
(\mathrm{N}=14)\end{array}$ \\
\hline Sensitivity & $\begin{array}{c}100 \% \\
(100 \%, 100 \%) \\
{[2 / 2]}\end{array}$ & $\begin{array}{c}67 \% \\
(13 \%, 100 \%) \\
{[2 / 3]}\end{array}$ & $\begin{array}{c}100 \% \\
(100 \%, 100 \%) \\
{[2 / 2]}\end{array}$ & $\begin{array}{c}100 \% \\
(100 \%, 100 \%) \\
{[3 / 3]}\end{array}$ & $\begin{array}{c}100 \% \\
(100 \%, 100 \%)\end{array}$ & $\begin{array}{c}83 \% \\
(42 \%, 97 \%)\end{array}$ \\
\hline Specificity & $\begin{array}{c}78 \% \\
(20 \%, 100 \%) \\
{[7 / 9]}\end{array}$ & $\begin{array}{c}55 \% \\
(25 \%, 84 \%) \\
{[6 / 11]}\end{array}$ & $\begin{array}{c}67 \% \\
(36 \%, 97 \%) \\
{[6 / 9]}\end{array}$ & $\begin{array}{c}27 \% \\
(1 \%, 54 \%) \\
{[3 / 11]}\end{array}$ & $\begin{array}{c}72 \% \\
(40 \%, 91 \%)\end{array}$ & $\begin{array}{c}41 \% \\
(20 \%, 66 \%)\end{array}$ \\
\hline $\begin{array}{l}\text { Overall Accuracy } \\
\text { (no. correct/total) }\end{array}$ & $\begin{array}{c}82 \% \\
(59 \%, 100 \%) \\
{[9 / 11]}\end{array}$ & $\begin{array}{c}57 \% \\
(31 \%, 83 \%) \\
{[8 / 14]}\end{array}$ & $\begin{array}{c}73 \% \\
(46 \%, 99 \%) \\
{[8 / 11]}\end{array}$ & $\begin{array}{c}43 \% \\
(17 \%, 69 \%) \\
{[6 / 14]}\end{array}$ & $\begin{array}{c}77 \% \\
(48 \%, 93 \%)\end{array}$ & $\begin{array}{c}50 \% \\
(29 \%, 71 \%)\end{array}$ \\
\hline
\end{tabular}

${ }^{a}$ Results presented as accuracy metrics $(95 \% \mathrm{Cl}$ ) and [positive or negative reads/total reads]. CEUS images were read by 2 independent readers. Results were considered per reader and as a combination of the 2 readers. For combined reader result, generalized estimating equations were used considering two readers' values as repeated measurements (or responses)

Abbreviations: CKD, chronic kidney disease; eGFR, estimated glomerular filtration rate

In the subgroup of patients who also had CT/MR, the number of patients who were CEUS negative but CT/ MR positive is smaller than the CEUS positive but CT/ MR negative group, suggesting that CEUS tends to assign a higher Bosniak category (or solid designation) than CT/MR (Fig. 4 and Fig. 5). This analysis does not take diagnosis into account and can therefore only be used to suggest that CEUS is more likely to call a lesion malignant than CT/MR and does not lead to conclusions about false positive or negative rates.

There are three possible explanations: First, ultrasound is more sensitive than CT/MR for detecting the presence of intravascular contrast. Unlike CT and MR agents, microbubble contrast agents remain intravascular: they are not filtered by glomeruli and do not extravasate into the interstitial space [41]. This may lead to more prominent internal enhancement with CEUS than CT/MR agents [22, 23]. Second, ultrasound has greater spatial resolution than CT/MR: in some cases (Fig. 4), cysts that appear simple on $\mathrm{CT} / \mathrm{MR}$ show internal features on ultrasound, including septations not seen on CT, resulting in a higher Bosniak grade using definitions based on CT or MR imaging. Better spatial and contrast resolution is an important advantage to examining lesions with CEUS; however, to reduce the false positive rate, the grading system will need to be modified to take this into account. Future, larger studies should be performed to develop a revised classification system [42]. Using objective measures of enhancement, similar to Hounsfield units for CT, may also improve CEUS accuracy. Furthermore, new CEUS imaging techniques such as super-resolution imaging [43], molecular imaging [44], and acoustic angiography [45] may further improve specificity to malignant lesions as they are translated into the clinic. The third possibility is that relative inexperience with CEUS compared to CT or MR leads to upstaging of kidney lesions.

Few studies have looked specifically at the use of CEUS for characterizing lesions in patients with CKD [46]. In this study, we examined patients with and without CKD. With more advanced CKD, overall accuracy declined, due largely to a decrease in specificity. While sensitivity only
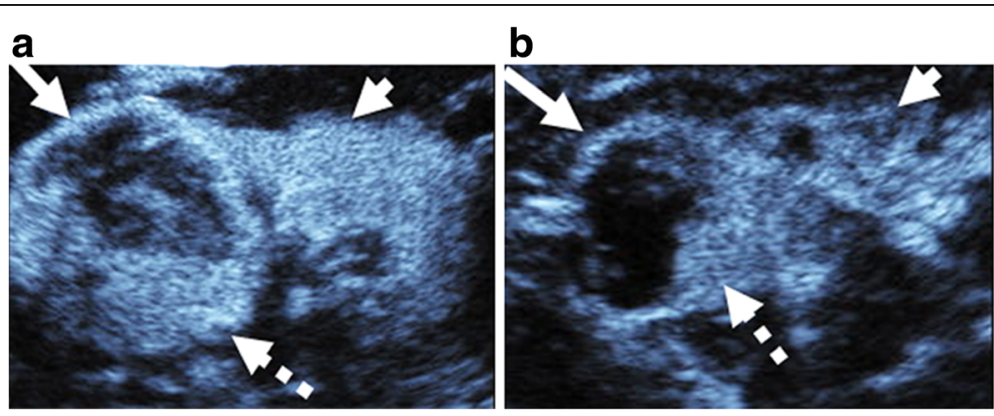

Fig. 3 Complex cystic lesions in 2 patients with chronic kidney disease of different severity. Sagittal CEUS images of two patients with complex cystic lesions, called Bosniak IV by both readers (long arrows). Mural nodularity (dashed arrows) is present in both cysts. Both lesions were confirmed as RCC on pathology. However, the 72-year old woman in 3a had early kidney disease, and the non-neoplastic kidney parenchyma (short arrow) in this case demonstrates homogeneous enhancement. $3 \mathbf{b}$ is a case of a 67-year old man with advanced kidney disease on dialysis in which the kidney parenchyma appears very different, demonstrating reduced, patchy enhancement (short arrow) 

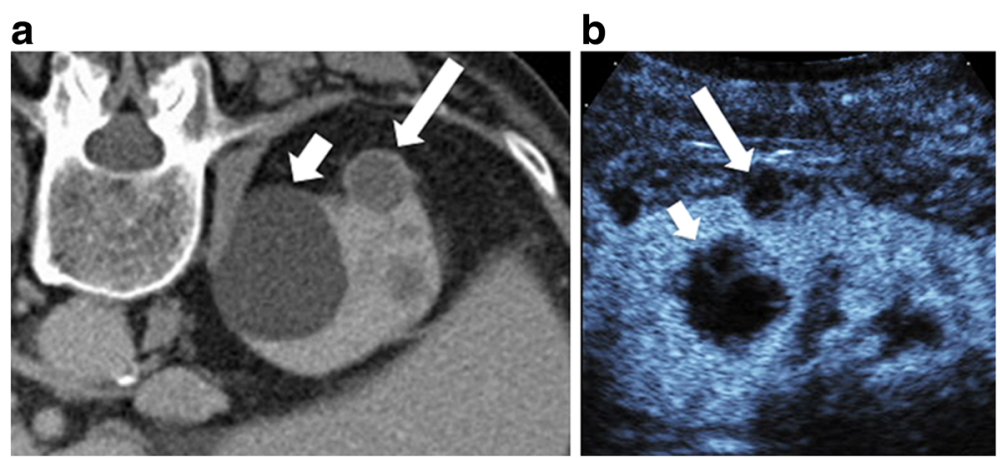

Fig. 4 Upstaging of cystic lesion on contrast-enhanced CT and CEUS due to greater special resolution. Contrast enhanced CT (4a) showing a smaller hyperdense cyst (long arrow) classified by readers as Bosniak II by one reader and IIF by the other in a 75 year old man. Adjacent to this is a large simple cyst (Bosniak I) (short arrow). On CEUS (4b), the smaller cyst (long arrow) shows enhancing internal septa and a solid component invisible on the CT that resulted in a Bosniak III classification. The larger cyst (short arrow) demonstrates internal features such as septations and wall irregularity that were also not visible on the CT. This illustrates the greater spatial resolution of CEUS compared to CT, and may explain why applying the Bosniak criteria to CEUS leads to upstaging

dropped from $100 \%$ for early CKD to $83 \%$ for advanced CKD, specificity dropped from $72 \%$ to $41 \%$. The reason for this requires further investigation, but we observed that while we were able to detect contrast enhancement in the uninvolved parenchyma for all patients, parenchymal enhancement was more heterogeneous and reduced in patients with CKD compared to non-CKD patients. We therefore hypothesize that a decrease in background parenchymal enhancement accentuates the difference in lesion enhancement compared to surrounding parenchyma. This abnormal parenchymal enhancement is even further pronounced in advanced CKD and may cause the lesion to stand out more, creating the perception that it is enhancing. This would be expected to primarily affect specificity, as the false-positive rate would increase with worsening CKD. With this in mind, we hypothesized that subjects with vascular CKD pathology (diabetes or hypertension) would have more false positives than nonvascular etiologies (cystic, glomerular, drug-related) but found no significant differences, likely due to small numbers. This should be explored further in larger populations. Nonetheless, the high sensitivity for ruling out malignancy in the CKD population suggests that this modality could be used for screening following noncontrast ultrasound or CT for patients in whom contrastenhanced CT/MR is contraindicated (Fig. 6). Larger studies are needed to confirm and extend our findings.

Based on our findings, we suggest that CEUS be considered to evaluate low to moderate-risk lesions in patients who have absolute or relative contra-indications to contrast CT or MR. If CEUS does not detect lesion enhancement, the patient may not need to undergo contrast CT or MR. If CEUS does detect lesion enhancement, further imaging should be considered. In addition, CEUS may have an application as a secondary test for lesions not definitively diagnosed by either contrast CT or MR. Due to the greater sensitivity to enhancement and special resolution, CEUS may be able to detect
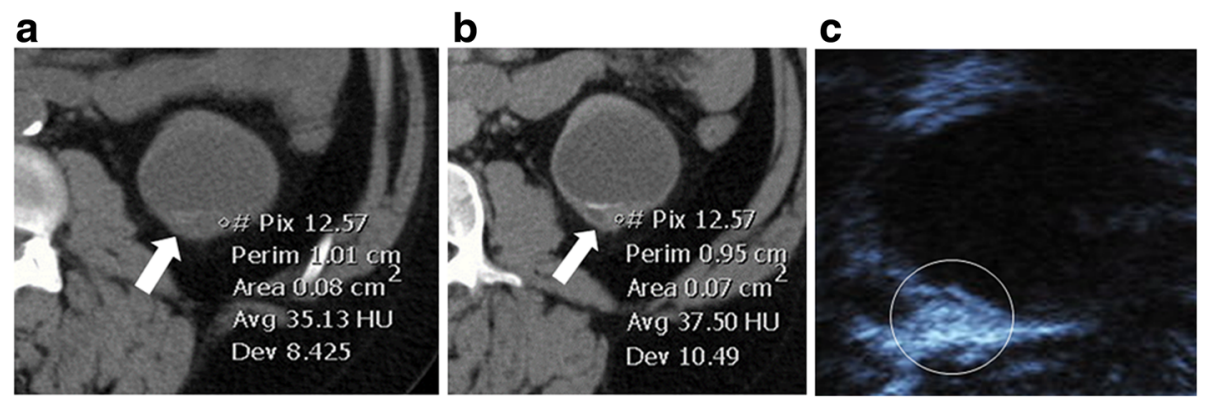

Fig. 5 Upstaging of cystic lesion on contrast-enhanced CT and CEUS due to greater contrast resolution. On the contrast-enhanced CT of a 44-year old patient with advanced kidney disease and kidney transplant, wall thickening is present (arrow). ROI shows no enhancement: the same Hounsfield unit measurements were seen pre- $(5 \mathbf{a}, 35 \mathrm{HU})$ and post-contrast $(5 \mathbf{b}, 37.5 \mathrm{HU})$. This was read by one radiologist as Bosniak II and the other as Bosniak III. On CEUS (5c), the thickened wall is irregular and clearly enhances. Both readers read the lesion (circled) one stage higher as Bosniak III and IV on CEUS. This illustrates that CEUS has greater contrast resolution than CT, and may explain why applying the Bosniak criteria to CEUS leads to upstaging 


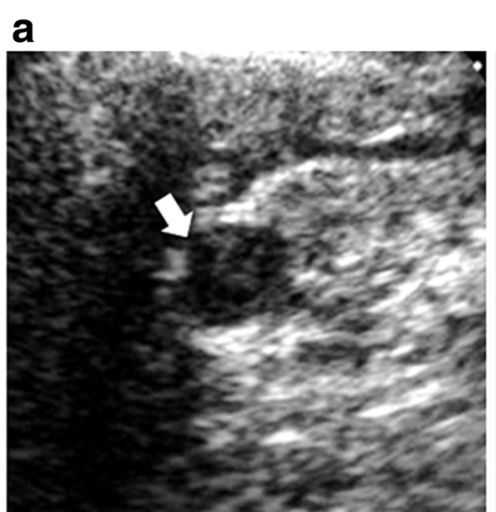

\section{b}

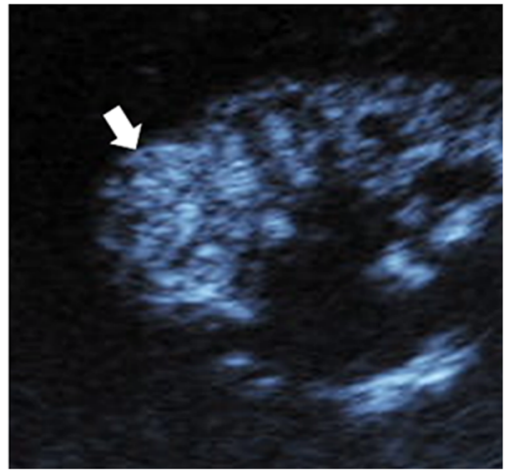

Fig. 6 Partly cystic lesion on CEUS compared to gray scale ultrasound. Gray scale longitudinal ultrasound (6a) shows an apparently partly cystic lesion within a strongly echogenic kidney in a 53-year old man with advanced CKD and hence contraindications to both contrast CT and MR. The lesion demonstrates intense homogeneous enhancement on CEUS (6b) and is larger but otherwise unchanged on 21-month follow-up imaging. It appeared partly cystic on grayscale ultrasound because it was surrounded by strongly echogenic CKD tissue. This case illustrates the difficulty of differentiating tumors from benign cysts on conventional ultrasound in the setting of advanced CKD

lesion enhancement where other modalities may not. This potential application should be evaluated in future studies.

Although CEUS is not yet FDA-approved for kidney imaging in the United States, it is increasingly used off label. Interpretation tools, such as the customized GUI, that address each component lesion characteristic of the Bosniak classification system, can assess reader consistency and inter-reader agreement. Observational training visits to institutions that have experience and familiarity with CEUS will improve implementation and interpretation accuracy.

This study has limitations. First, this is a pilot study with a relative small size population from a single center. Due to the small size, meaningful subgroup analyses, such as those at each stage of CKD and those with cystic vs. solid lesions, was not possible. Because of this limitation, the conclusions drawn are preliminary in nature and should be interpreted with caution. Future, larger, multicenter studies are needed to determine the true accuracy of CEUS among individuals with diverse stages of CKD. Second, the blinded readers in this study were academic genitourinary radiologists with fellowship training in ultrasound. They underwent a preliminary training session and performed CEUS literature review, but neither had prior experience with CEUS. However, their background is not atypical as physicians are now becoming more familiar with CEUS in the United States, particularly in academic centers. Reassuringly, the sensitivities observed were similar to those reported by more experienced centers [34]. As there is little available formal training, development of a training program and experience with interpretation of CEUS is needed before widespread implementation. Additionally, because CEUS is a perfusion study, applying objective perfusion parameters could increase accuracy. Third, the reference standard of tissue diagnosis was not available for all patients because many indeterminate lesions underwent imaging surveillance rather than biopsy or surgery. A minimum of one-year follow-up was accepted, but the ideal follow-up period is $3-5$ years, depending on lesion complexity, as some renal cancers are slow growing. Until longer follow-up data on more patients is obtained, malignancy cannot be ruled out with a Bosniak IIF lesion, and these patients would still need to be followed, as is the case with contrast CT and MR. Fourth, both cystic and solid lesions were included. In order to more accurately determine accuracy and potential modifications to Bosniak criteria for CEUS in a CKD population, future studies will need to include primarily cystic lesions. Fifth, the referral source for the two groups, non-CKD and CKD, was different. This introduces potential bias since the non-CKD group came primarily from urology clinic where patients were referred for evaluation of highly suspicious lesions (i.e. likely higher risk lesions), and the CKD group came primarily from non-urologists who were longitudinally following indeterminate lesions (i.e. likely lower risk lesions). Lastly, as with any ultrasound study, obesity, lesion location and motion artifact from breathing are technical limitations.

\section{Conclusion}

The traditional Bosniak classification system was developed to classify kidney cystic lesions based on contrast-enhanced CT characteristics We adapted this classification system for B-mode US/CEUS and found that it has potential as a test to exclude malignancy based on a sensitivity comparable to CT/MRI, but it does not yet have good specificity. Low specificity might be attributed to the greater spatial resolution and contrast detection with CEUS compared to 
contrast-enhanced CT, leading to upstaging of lesions. It may also be attributed to lack of experience interpreting CEUS studies. Our results suggest that with further refinement of a classification system and more training, CEUS may be an alternative to contrast CT or MR for characterizing indeterminate kidney lesions, particularly in patients with CKD or other contraindications to contrast CT or MR. However, if the traditional Bosniak criteria are used for CEUS, malignancy can still be excluded but a positive CEUS result will require further diagnostic evaluation due to its low specificity. Improved specificity may be accomplished with training programs for radiologists and revisions to the Bosniak criteria, potentially adopting a classification scheme proposed by Barr et al. [24] which incorporates both solid and cystic lesions. Larger studies focused on cystic lesions, particularly in patients with later stages of CKD are needed to confirm and extend our findings and inform classification criteria revisions.

\section{Additional files}

Additional file 1: Table S1. Bosniak criteria adapted to CEUS. Adaptations in bold. Table S2. Overall accuracy of CEUS lesion designation by co-morbid conditions in patients with CKD. Table S3. Accuracy of CEUS in patients with purely solid lesions and cystic lesions or lesions with a cystic component. Table S4. Accuracy of CEUS compared to tissue diagnosis or follow-up imaging in patients with lesions $<3 \mathrm{~cm}$ and $\geq 3 \mathrm{~cm}$ in patients with and without CKD. (DOCX $15 \mathrm{~kb})$

Additional file 2: Figure S1. The custom designed graphical user interface used by the readers to interpret CEUS images (PNG $40123 \mathrm{~kb}$ )

Additional file 3: Imaging parameters. (DOCX $13 \mathrm{~kb}$ )

\section{Acknowledgments}

The authors would like to thank Gayle Grigson for her indispensable services during conduction of the study, Ismayil Guracar at Siemens Medical Solutions for his technical advice, David Cosgrove for his advice and guidance, and Jennifer E. Flythe, MD for her invaluable advice, mentoring and editing.

\section{Funding}

Support for this work was provided by NC TraCS at the University of North Carolina at Chapel Hill, Lineberger Comprehensive Cancer Center Core Grant and $\mathrm{NIH}$ grant K24CA172355. No specific roles were fulfilled by any funding agency.

\section{Availability of data and materials}

The datasets used and/or analyzed during the current study are available from the corresponding author on reasonable request.

\section{Authors' contributions}

EHC, WKC, and WKR were responsible for the integrity of entire study. WKC and WKR contributed to study design. EHC and WKR were responsible for patient recruitment. EHC, WKC, WKR, PAD, SKK, and LBM participated in acquisition of images. JRF and EA performed blinded image interpretations. JF and JIK performed statistical analysis. EHC was primarily responsible for manuscript drafting, and WKR, WKC, SKK, PAD, JRF, EA participated in manuscript revision for important intellectual content. All authors gave manuscript final version approval. All authors agree to be accountable for all aspects of the work in ensuring that questions related to the accuracy or integrity of any part of the work are appropriately investigated and resolved. All authors read and approved the final manuscript.

\section{Ethics approval and consent to participate}

This study was performed in compliance with the policies related to the use of human subjects of the Biomedical Institutional Review Board, study number 12-2314, and in compliance with the provisions of the Health Insurance Portability and Accountability Act (HIPAA). The committee members that approved the study, and their affiliations are: J. Herbert Patterson (Pharmacy), Beth Boyea (Reproductive Genetics), Ruth Ann Bark (Clinical Research Management), John Buse (Diabetes), David Edwards (Prisoner Representative), Stuart H. Gold (Oncology), Howard Kehrl (General Medicine), Paris Laliberte (Latino Health), Marjorie Land (unspecified), Steven Lichtman (Pediatrics), Robert Matthews (Anesthesiology, Nursing).

All procedures performed in studies involving human participants were in accordance with the ethical standards of the institutional and/or national research committee and with the 1964 Helsinki declaration and its later amendments or comparable ethical standards. Informed consent was obtained from all individual participants included in the study.

\section{Consent for publication}

All individuals who have data (images) presented have consented to have their data submitted for publication.

\section{Competing interests}

The authors declare that they have no competing interests.

\section{Publisher's Note}

Springer Nature remains neutral with regard to jurisdictional claims in published maps and institutional affiliations.

\section{Author details}

'University of North Carolina, 7024 Burnett Womack, CB 7155, Chapel Hill, NC 27599, USA. ${ }^{2}$ Present address: University of Texas Southwestern at Dallas, 5323 Harry Hines Boulevard, Dallas, TX 75390-8827, USA. ${ }^{3}$ Present address: Department of Medicine, Division of Hematology and Oncology, Vanderbilt University, 777 Preston Research Building, Nashville, TN 37232, USA. ${ }^{4}$ Diagnostic Radiology, Abdominal Imaging Section, The University of Texas MD Anderson Cancer Center, Unit 1473 FCT15.5092, 1400 Pressler Street, Houston, TX 77030, USA. ${ }^{5}$ Department of Radiology, University of North Carolina at Chapel Hill, CB 7510, Chapel Hill, NC 27599, USA. ${ }^{6}$ Department of Biostatistics, University of North Carolina, 3101 McGavran-Greenberg Hall, CB

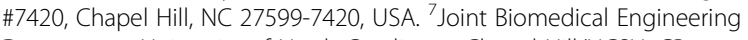
Department, University of North Carolina at Chapel Hill/NCSU, CB 7575, Chapel Hill, NC 27599, USA. ${ }^{8}$ University of North Carolina, Lineberger Cancer Center, NC 27599, Chapel Hill, USA.

Received: 1 May 2017 Accepted: 28 July 2017

Published online: 09 August 2017

References

1. Society AC. Cancer Fact \& Figures 2017. Atlanta: American Cancer Society; 2017.

2. Organ M, Jewett M, Basiuk J, Morash C, Pautler S, Siemens DR, Tanguay S, Gleave M, Drachenberg D, Chow R, et al. Growth kinetics of small renal masses: a prospective analysis from the renal cell carcinoma consortium of Canada. Can Urol Assoc J. 2014;8(1-2):24-7.

3. Pandharipande PV, Gervais DA, Hartman Rl, Harisinghani MG, Feldman AS, Mueller PR, Gazelle GS. Renal mass biopsy to guide treatment decisions for small incidental renal tumors: a cost-effectiveness analysis. Radiology. 2010; 256(3):836-46.

4. Tsivian M, Rampersaud EN Jr, del Pilar Laguna Pes M, Joniau S, Leveillee RJ, Shingleton WB, Aron M, Kim CY, AM DM, Desai MM, et al. Small renal mass biopsy-how, what and when: report from an international consensus panel. BJU Int. 2014;113(6):854-63.

5. Borghesi M, Brunocilla E, Volpe A, Dababneh H, Pultrone CV, Vagnoni V, La Manna G, Porreca A, Martorana G, Schiavina R. Active surveillance for clinically localized renal tumors: an updated review of current indications and clinical outcomes. Int J Urol. 2015;22(5):432-8.

6. Bahouth Z, Halachmi S, Meyer G, Avitan O, Moskovitz B, Nativ O. The natural history and predictors for intervention in patients with small renal mass undergoing active surveillance. Adv Urol. 2015;2015:692014.

7. Pierorazio PM, Johnson MH, Ball MW, Gorin MA, Trock BJ, Chang P, Wagner AA, McKiernan JM, Allaf ME. Five-year analysis of a multi-institutional 
prospective clinical trial of delayed intervention and surveillance for small renal masses: the DISSRM registry. Eur Urol. 2015;68(3):408-15.

8. Sahni VA, Silverman SG. Imaging management of incidentally detected small renal masses. Semin Intervent Radiol. 2014;31(1):9-19.

9. Bosniak MA. The use of the Bosniak classification system for renal cysts and cystic tumors. J Urol. 1997;157(5):1852-3.

10. Bosniak MA. The Bosniak renal cyst classification: 25 years later. Radiology. 2012;262(3):781-5

11. Kanda T, Fukusato T, Matsuda M, Toyoda K, Oba H, Kotoku J, Haruyama T, Kitajima K, Furui S. Gadolinium-based Contrast Agent Accumulates in the Brain Even in Subjects without Severe Renal Dysfunction: Evaluation of Autopsy Brain Specimens with Inductively Coupled Plasma Mass Spectroscopy. Radiology. 2015;142690:1-5.

12. McDonald RJ, McDonald JS, Kallmes DF, Jentoft ME, Murray DL, Thielen KR, Williamson EE, Eckel LJ. Intracranial Gadolinium Deposition after Contrast-enhanced MR Imaging. Radiology 2015;150025.

13. Olchowy C, Cebulski K, Lasecki M, Chaber R, Olchowy A, Kalwak K, Zaleska-Dorobisz $\mathrm{U}$. The presence of the gadolinium-based contrast agent depositions in the brain and symptoms of gadolinium neurotoxicity - a systematic review. PLoS One. 2017;12(2):e0171704.

14. Hofmann JN, Corley DA, Zhao WK, Colt JS, Shuch B, Chow WH, Purdue MP. Chronic kidney disease and risk of renal cell carcinoma: differences by race. Epidemiology. 2015;26(1):59-67.

15. Christensson A, Savage C, Sjoberg DD, Cronin AM, O'Brien MF, Lowrance W, Nilsson PM, Vickers AJ, Russo P, Lilja H. Association of cancer with moderately impaired renal function at baseline in a large, representative, population-based cohort followed for up to 30 years. Int J Cancer. 2013; 133(6):1452-8.

16. Lowrance WT, Ordonez J, Udaltsova N, Russo P, Go AS. CKD and the risk of incident cancer. J Am Soc Nephrol. 2014;25(10):2327-34.

17. ACR Manual on Contrast Media. In., 10 edn; 2016.

18. Cantisani V, Bertolotto M, Weskott HP, Romanini L, Grazhdani H, Passamonti M, Drudi FM, Malpassini F, Isidori A, Meloni FM, et al. Growing indications for CEUS: the kidney, testis, lymph nodes, thyroid, prostate, and small bowel. Eur J Radiol. 2015;84(9):1675-84.

19. Qiao JJ, Yu J, Yu Z, Li N, Song C, Li M. Contrast-enhanced ultrasonography in differential diagnosis of benign and malignant ovarian tumors. PLoS One. 2015;10(3):e0118872

20. Sparchez Z, Radu P, Sparchez M, Crisan N, Kacso G, Petrut B. Contrast enhanced ultrasound of renal masses. A reappraisal of EFSUMB recommendations and possible emerging applications. Med Ultrason. 2015; 17(2):219-26.

21. Park BK, Kim B, Kim SH, Ko K, Lee HM, Choi HY. Assessment of cystic renal masses based on Bosniak classification: comparison of $C T$ and contrastenhanced US. Eur J Radiol. 2007;61(2):310-4.

22. Quaia E, Bertolotto M, Cioffi V, Rossi A, Baratella E, Pizzolato R, Cov MA. Comparison of contrast-enhanced sonography with unenhanced sonography and contrast-enhanced CT in the diagnosis of malignancy in complex cystic renal masses. AJR Am J Roentgenol. 2008;191(4):1239-49.

23. Chen $Y$, Wu N, Xue T, Hao Y, Dai J. Comparison of contrast-enhanced sonography with MRI in the diagnosis of complex cystic renal masses. J Clin Ultrasound: JCU. 2014. p. 1-7.

24. Barr RG, Peterson C, Hindi A. Evaluation of indeterminate renal masses with contrast-enhanced US: a diagnostic performance study. Radiology. 2014; 271(1):133-42.

25. Ascenti G, Mazziotti S, Zimbaro G, Settineri N, Magno C, Melloni D, Caruso R, Scribano E. Complex cystic renal masses: characterization with contrast-enhanced US. Radiology. 2007;243(1):158-65.

26. Bertolotto M, Cicero C, Perrone R, Degrassi F, Cacciato F, Cova MA. Renal masses with equivocal enhancement at CT: characterization with contrast-enhanced ultrasound. AJR Am J Roentgenol. 2015;204(5):W557-65.

27. Sanz E, Hevia V, Gomez V, Alvarez S, Fabuel JJ, Martinez L, Rodriguez-Patron R, Gonzalez-Gordaliza C, Burgos FJ. Renal complex cystic masses: usefulness of contrast-enhanced ultrasound (CEUS) in their assessment and its agreement with computed tomography. Curr Urol Rep. 2016;17(12):89.

28. Greis C. Summary of technical principles of contrast sonography and future perspectives. Radiologe. 2011;51(6):456-61.

29. Greis C. Ultrasound contrast agents as markers of vascularity and microcirculation. Clin Hemorheol Microcirc. 2009;43(1-2):1-9.

30. Lindner JR, Song J, Jayaweera AR, Sklenar J, Kaul S. Microvascular rheology of Definity microbubbles after intra-arterial and intravenous administration.
J American Society of Echocardiography: official publication of the American Society of Echocardiography. 2002;15(5):396-403.

31. Lindner JR, Wei K. Contrast echocardiography. Curr Probl Cardiol. 2002; 27(11):454-519.

32. Piscaglia F, Bolondi L. Italian Society for Ultrasound in M, biology study group on ultrasound contrast a. The safety of Sonovue in abdominal applications: retrospective analysis of 23188 investigations. Ultrasound Med Biol. 2006:32(9):1369-75.

33. Wei K, Mulvagh SL, Carson L, Davidoff R, Gabriel R, Grimm RA, Wilson S, Fane L, Herzog CA, Zoghbi WA, et al. The safety of deFinity and Optison for ultrasound image enhancement: a retrospective analysis of 78,383 administered contrast doses. J American Society of Echocardiography: official publication of the American Society of Echocardiography. 2008; 21(11):1202-6

34. Sawhney S, Wilson SR. Can Ultrasound With Contrast Enhancement Replace Nonenhanced Computed Tomography Scans in Patients With Contraindication to Computed Tomography Contrast Agents? Ultrasound Q. 2017;

35. Girometti R, Stocca T, Serena E, Granata A, Bertolotto M. Impact of contrast-enhanced ultrasound in patients with renal function impairment. World J Radiol. 2017;9(1):10-6.

36. Claudon M, Dietrich CF, Choi Bl, Cosgrove DO, Kudo M, Nolsoe CP, Piscaglia F, Wilson SR, Barr RG, Chammas MC, et al. Guidelines and good clinical practice recommendations for contrast enhanced ultrasound (CEUS) in the liver - update 2012: a WFUMB-EFSUMB initiative in cooperation with representatives of AFSUMB, AIUM, ASUM. FLAUS and ICUS Ultrasound Med Biol. 2013:39(2):187-210.

37. Yan J, Fine J. Estimating equations for association structures. Stat Med. 2004 23(6):859-74. discussion 75-7,79-80

38. Amin MB, Amin MB, Tamboli P, Javidan J, Stricker $H$, de-Peralta Venturina $M$ Deshpande A, Menon M. Prognostic impact of histologic subtyping of adult renal epithelial neoplasms: an experience of 405 cases. Am J Surg Pathol. 2002;26(3):281-91.

39. Tickoo SK, dePeralta-Venturina MN, Harik LR, Worcester HD, Salama ME, Young AN, Moch H, Amin MB. Spectrum of epithelial neoplasms in endstage renal disease: an experience from 66 tumor-bearing kidneys with emphasis on histologic patterns distinct from those in sporadic adult renal neoplasia. Am J Surg Pathol. 2006;30(2):141-53.

40. Chang EH, Chong WK, Kasoji S, Dayton PA, Rathmell WK. Management of Indeterminate Cystic Kidney Lesions: Review of Contrast-Enhanced Ultrasound as a Diagnostic Tool. Urology. 2015;

41. Kalantarinia K, Okusa MD. Ultrasound contrast agents in the study of kidney function in health and disease. Drug Discov Today Disease Mech. 2007;4(3): 153-8.

42. Barr RG. Is there a need to modify the Bosniak renal mass classification with the addition of contrast-enhanced Sonography? J Ultrasound Med. 2017; 36(5):865-8.

43. Errico C, Pierre J, Pezet S, Desailly Y, Lenkei Z, Couture O, Tanter M. Ultrafast ultrasound localization microscopy for deep super-resolution vascular imaging. Nature. 2015;527(7579):499-502.

44. Kircher MF, Willmann JK. Molecular body imaging: MR imaging, CT, and US. Part II Appl Radiol. 2012;264(2):349-68.

45. Gessner RC, Aylward SR, Dayton PA. Mapping microvasculature with acoustic angiography yields quantifiable differences between healthy and tumor-bearing tissue volumes in a rodent model. Radiology. 2012;264(3): 733-40.

46. Paudice N, Zanazzi M, Agostini S, Bertelli E, Caroti L, Carta P, Moscarelli L, Tsalouchos A, Salvadori M, Bertoni E. Contrast-enhanced ultrasound assessment of complex cystic lesions in renal transplant recipients with acquired cystic kidney disease: preliminary experience. Transplant Proc. 2012;44(7):1928-9. 